\title{
Análisis Comparativo en Muestras de Guadua Angustifolia Kunth Solicitadas a Compresión Paralela a la Fibra
}

\author{
Comparative Analysis in Guadua angustifolia Kunth Samples \\ Solicited to Compression Parallel to the Fiber
}

Caori Takeuchi, Mauricio Duarte y Wilson Erazo

\begin{abstract}
Resumen
Se presenta una comparación entre los resultados de resistencia a compresión y valores caracteristicos obtenidos en diferentes investigaciones realizadas en muestras de Guadua angustífolia Kunth, solicitadas a compresión paralela a la fibra procedentes de diversas partes de Colombia, con los encontrados en el trabajo de investigación "Resistencia a la compresión paralela a la fibra y determinación del módulo de elasticidad de la Guadua angustífolia del municipio de Pitalito-Huila", En este trabajo, primero en su tipo realizado en el departamento del Huila Colombia, se aplicó la metodología de la Norma Técnica Colombiana NTC 5525 y los protocolos de la Universidad Nacional de Colombia sede Bogotá (2008) "Corte de culmos y preparación de probetas, determinación de la resistencia a compresión paralela a la fibra y medición del contenido de humedad para la guadua angustifolia Kunth". Se realizó una clasificación de la información recopilada, teniendo en cuenta la ubicación y la metodología implementada. Se observó que la Guadua angustifolia del departamento del Huila presentó un buen desempeño, con valores de resistencia última de $37,83 \mathrm{Mpa}$, valor característico promedio de $28,49 \mathrm{Mpa}$ para contenidos de humedad promedio de $86,16 \%$.
\end{abstract}

Palabras clave: Comparación; Guadua angustifolia; compresión paralela a la fibra.

\begin{abstract}
This article presents a comparison between the results of strength and characteristic values found in different studies performed on Guadua angustifolia Kunth samples under compression parallel to the fiber taken from different parts of Colombia, with the results from research project "Compressive strength parallel to the fiber and determination of modulus elasticity of Guadua angustifolia of the municipality of Pitalito-Huila". This work, first of its type carried in the department of Huila Colombia, followed the procedures of the standard NTC 5525 and the protocols "Cutting culms and preparation of samples, for the determination of the compressive strength parallel to the fiber and measurement of moisture content for Guadua angustifolia Kunth samples" of the Universidad Nacional de Colombia in Bogotá (2008). The information collected was classified, considering the location and the methodology used. The Guadua angustifolia of the department of Huila presented a good performance, with values of ultimate strength of $37.83 \mathrm{MPa}, 28.49 \mathrm{Mpa}$ in characteristic value with moisture content average of $86.16 \%$.
\end{abstract}

Keywords: Comparison; Guadua angustifolia; compressive strength parallel to the fiber.

1 Magister en Estructuras. Docente Universidad Nacional de Colombia sede Bogotá. cptakeuchit@unal.edu.co

2 Magister en Ingeniería Civil. Docente Universidad Surcolombiana. Av. Pastrana Borrero carrera $L^{1}$ Neiva. maduto@usco.edu.co

3 Ingeniero Agricola. Universidad Surcolombiana. Av. Pastrana Borrero carrera 1² Neiva. wjec89@gmail.com 


\section{Introducción}

El sur del departamento del Huila Colombia posee características óptimas desde el punto de vista ambiental para el crecimiento y desarrollo adecuado de la especie Guadua angustifolia Kunth. En esta zona cafetera al sur del departamento, se encuentran los municipios de Pitalito y Timaná, que fueron seleccionados como lugar objeto de investigación en el trabajo "Resistencia a la compresión paralela a la fibra y determinación del módulo de elasticidad de la Guadua angustifolia del municipio de Pitalito-Huila". El rango de altitud para estos municipios es de 1000 a 2000 m.s.n.m, con temperaturas que pueden variar desde 18 hasta $24^{\circ} \mathrm{C}$. En lugares especificos de estos municipios, se presentan las condiciones definidas por Garcia (2004) como de mayor favorabilidad para las variables de calidad: dureza, humedad, resistencia a la compresión, resistencia al corte, densidad básica y longitud de canutos; con altitudes entre $\operatorname{los} 1200$ y $1500 \mathrm{~m} \cdot \mathrm{s} . n \mathrm{~m}$, temperaturas entre 20 y $22^{\circ} \mathrm{C}$ y pendientes entre 20 y $30^{\circ}$.

En esta zona del pais, la Guadua angustifolia Kunth es utilizada principalmente para la adecuación de la infraestructura destinada al beneficio del café, como beneficiaderos y secadores solares; su uso en estructuras de mayor tamaño y complejidad es poco frecuente por la falta de atención hacia este recurso y el desconocimiento de sus propiedades fisico-mecánicas; conviene mencionar que previo a la elaboración del trabajo anteriormente mencionado, el departamento no contaba con estudios relacionados con las propiedades mecánicas de la guadua. Lo anterior, limita entre otras cosas el aprovechamiento, la producción y la tecnificación de este material.

En el proyecto referido se determinaron: la resistencia última a la compresión paralela a la fibra, los valores caracteristicos, los esfuerzos admisibles, los contenidos de humedad, los módulos de elasticidad y la densidad básica en probetas de guadua angustifolia. Algunas de estas variables se habian investigado con anterioridad en estudios como los desarrollados por Martin y Mateus (1981), Martinez (1992), López y Silva (2000), Durán y Uribe (2002), García (2004), González (2006), González et al (2008), Lamus (2008), Rivera (2008), Lozano et al (2010) y Muñoz (2011), en muestras representativas de guadua angustifolia de los departamentos de Caldas, Cundinamarca, Quindio, Risaralda y Valle del Cauca, los cuales significaron un aporte importante para la industria y el sector de la construcción principalmente. La transcendencia de estos y otros estudios, se puede ver reflejada en la reglamentación de normas como las NTC 5300, 5301, 5407 y 5525, y el capitulo G.12 Estructuras de Guadua en el actual Reglamento Colombiano de Construcción Sismo Resistente (NSR-10), normas que se derivan además de estándares internacionales ISO 22156, 22157.

Para efectos de comparación entre los resultados en resistencia a la compresión paralela a la fibra de la guadua del departamento del Huila con los resultados de las investigaciones anteriormente mencionadas, se debe considerar principalmente los valores característicos, contenidos de humedad y la metodologia empleada; los valores característicos, por tratarse de valores de referencia; la variación del contenido de humedad, porque afecta la resistencia de la guadua; y la metodología implementada, porque se han realizado ajustes con el tiempo en lo que corresponde a toma de muestras y ejecución de ensayos, lo cual podria explicar las diferencias entre los resultados de las diferentes investigaciones.

En orden cronológico se sabe que los primeros estándares para la determinación de las propiedades fisicas y mecánicas del bambú fueron internacionales: "Inbar standard for determination of physical and mechanical properties on bamboo" y "Laboratory manual on testing methods for determination of physical and mechanical properties on bamboo" en el año 1999, seguido de la norma ISO 22157 en el 2004; más tarde a nivel nacional, especificamente para el bambú guadua, surgió la Norma Técnica Colombiana NTC 5525 en el 2008 y por último en el 2010 el capitulo G.12 de la NSR-10. En el proyecto tomado como referencia "Resistencia a la compresión paralela a la fibra y determinación del módulo de elasticidad de la guadua angustifolia del municipio de PitalitoHuila", se implementaron los estándares nacionales y los protocolos de la Universidad Nacional de Colombia sede Bogotá (2008). "Corte de culmos y preparación de probetas, determinación de la resistencia a compresión paralela a la fibra y medición del contenido de humedad para la Guadua angustifolia Kunth". 


\section{Metodología}

En el proyecto "Resistencia a la compresión paralela a la fibra y determinación del módulo de elasticidad de la Guadua angustifolia del municipio de Pitalito-Huila" tomado como referencia, se seleccionaron culmos de Guadua angustifolia como material objeto de investigación, procedente de cuatro plantaciones ubicadas en el municipio de Pitalito y una en el municipio de Timaná, departamento del Huila, Colombia. La localización de cada una garantizó la representatividad de la zona de estudio (tabla 1).

Tabla 1. Localización de las zonas en los mumicipios de Pitalito y Timaná

\begin{tabular}{l|c|c|c|c|c}
\hline & & & \multicolumn{2}{|c|}{ Coordenadas Magna-Sirgas } & \\
\hline Predio & Vereda & Municipio & Latitud (N) & Longitud (O) & $\begin{array}{c}\text { Altitud } \\
\text { (m.s.n.m) }\end{array}$ \\
\hline A: La Vega & Pantanos & Timaná & $1^{\circ} 54^{\prime} 19.80^{\prime \prime}$ & $75^{\circ} 58^{\prime} 02.80^{\prime \prime}$ & 1253 \\
\hline B: La Esperanza & San Francisco & Pitalito & $1^{\circ} 50^{\prime \prime} 06.00^{\prime \prime}$ & $76^{\circ} 06^{\prime} 57.40^{\prime \prime}$ & 1313 \\
\hline C: La Dalia-Limón & Palmarito & Pitalito & $1^{\circ} 47^{\prime} 13.80^{\prime \prime}$ & $76^{\circ} 04^{\prime} 03.40^{\prime \prime}$ & 1294 \\
\hline D: Sena Yamboró & Aguadas & Pitalito & $1^{\circ} 53^{\prime \prime} 36.00^{\prime \prime}$ & $76^{\circ} 05^{\prime} 25.10^{\prime \prime}$ & 1331 \\
\hline E: Villa María & Zanjones & Pitalito & $1^{\circ} 50^{\prime} 26.11^{\prime \prime}$ & $76^{\circ} 01^{\prime} 58.31^{\prime \prime}$ & 1279 \\
\hline
\end{tabular}

Los culmos fueron cortados y transportados en tres secciones: inferior, media y superior para ser caracterizados fisicamente en la Universidad Nacional de Colombia sede Bogotá. Las probetas extraídas de cada una de las secciones fueron igualmente caracterizadas (Figura 1) y posteriormente ensayadas a compresión paralela a la fibra (Figura 2) hasta la falla para obtener la resistencia última. Las 132 probetas se obtuvieron de forma aleatoria, cada una por sección de culmo. La selección de probetas con nudo y sin nudo se realizó de la misma forma. Se determinó para cada una, el contenido de humedad al momento de la prueba. Las herramientas y equipos utilizados durante todo el proceso se muestran a continuación

- Equipo de carga a compresión FORNEY con capacidad de carga de $500 \mathrm{Lb}$

- Balanza digital BJ 4100D PRECISA, con exactitud de 0,1g.

- Balanza digital OHAUS (PionnerTM), con exactitud de $0,01 \mathrm{~g}$.

- Horno. Control electrónico con temperatura.

- Horno eléctrico marca THELCO (Thermoscientific).

- Acolilladora marca PROTECH.

- Pie de rey.

Para los cortes, la caracterización de las probetas y los culmos, y las pruebas de resistencia y el contenido de humedad, se aplicaron las metodologias propuestas por las siguientes normas y protocolos:

- Norma unificada para el manejo y aprovechamiento de la Guadua, (Carder, 2002).

- Ley forestal 1021 de 2006

- ISO/TC $165 \mathrm{~N} 315,2001$. "Laboratory manual on testing methods for determination of physical and mechanical properties of bamboo"

- NTC5300 "Cosecha y postcosecha del culmo de Guadua angustifolia Kunth" (Icontec, 2008).

- NTC5525 "Métodos de ensayo para determinar las propiedades físicas y mecánicas de la Guadua" (Icontec, 2007).

- Reglamento Colombiano de Construcciones Sismo-Resistente NSR10, 2010. Título G "Estructuras de madera y estructuras de Guadua". Bogotá D.C

- Protocolos de la Universidad Nacional de Colombia sede Bogotá (2008). "Corte de culmos y probetas, determinación de la resistencia a compresión paralela a la fibra, medición del contenido de humedad para la Guadua angustifolia Kunth". 


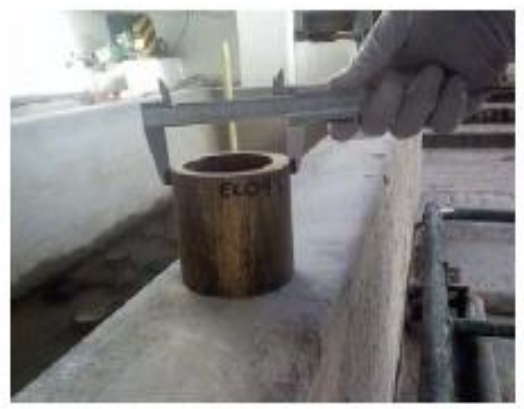

Figura l. Caracterización fisica de probetas

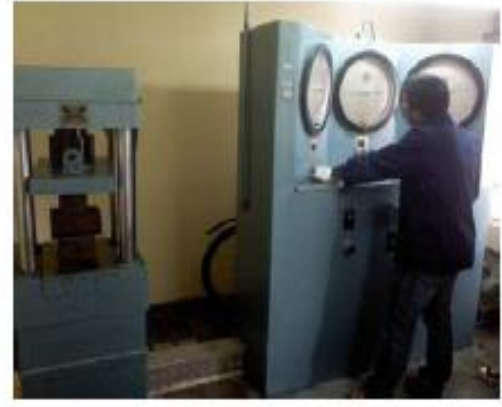

Figura 2. Ensayos de resistencia a la compresión paralela a la fibra

Los datos de resistencia última a la compresión paralela a la fibra y contenidos de humedad, se analizaron de manera exploratoria para excluir valores atípicos, se determinaron valores promedios, mínimos y máximos, y medidas de dispersión mediante análisis descriptivo, con el fin de aplicarlos en las ecuaciones 1 y 2 para el cálculo de valores caracteristicos y percentil 5 respectivamente.

Ecuación 1: Valor Característico segín G.12.7.4 - NSR-10

Dónde:

$$
\mathrm{f}_{\mathrm{kc}}=\mathrm{f}_{0,05 \mathrm{i}}\left[1-\frac{2,7 \frac{\mathrm{i}}{\mathrm{m}}}{\sqrt{\mathrm{n}}}\right] \text { Ecu. }(1)
$$

fo.0sc : Valor correspondiente al percentil 5 de los datos (Mpa)

$\mathrm{m}^{\prime} \quad$ : Valor promedio de los datos (Mpa)

s : Desviación estándar de los datos

n : Número de ensayos (por lo menos 20)

Ecuación 2: Percentil 5 segin (G.A.1 - NSR-10):

$$
\mathrm{F}_{\mathrm{c} 0,05}=\overline{\mathrm{F}}_{\mathrm{c}}\left(1-1,645 \mathrm{COV}_{\mathrm{c}}\right) \text { Ecu. }(2)
$$

Dónde:

\section{$\overline{\mathrm{F}}_{c} \quad$ : Esfuerzo básico promedio (Mpa)}

COVc : Coeficiente de variación de esfuerzos

Para establecer comparaciones con otros estudios, se recopiló la información relacionada con el proyecto antes referido y con los obtenidos en investigaciones de las propiedades fisicas y mecánicas en muestras de guadua angustifolia (rolliza) procedentes de los departamentos de Caldas, Cundinamarca, Quindio, Risaralda y Valle del Cauca.

La información recopilada fue clasificada por procedencia y metodologia aplicada. En relación con la procedencia, la información se clasificó por regiones debido al cambio en la posición geográfica y las condiciones de sitio que podrian incidir en la calidad de la guadua. Con respecto a la segunda, se encontraron metodologias distintas para la selección del material, preparación de probetas y ejecución de ensayos. La razón principal por la que se presentan estas diferencias, están en la realización de estudios previos y posteriores a las publicaciones de las normas ISO 22157 en el 2004 y las Norma Técnica Colombiana NTC 5525 en el 2007. 


\section{Resultados}

Se presenta a continuación los resultados del análisis estadistico descriptivo realizado en el proyecto "Resistencia a la compresión paralela a la fibra y determinación del módulo de elasticidad de la Guadua angustifolia del municipio de Pitalito-Huila". Se muestran, los resultados de otros estudios de acuerdo con la clasificación realizada de la información y por último el análisis comparativo para resistencia última y valores característicos.

\subsection{Guadua del departamento del Huila}

En el análisis exploratorio se encontraron valores atipicos de resistencia última por lo que fueron excluidos. En total se analizaron 130 probetas con un contenido de humedad promedio de $86,16 \%$, clasificadas según el lugar de corte en los culmos. La información necesaria para llegar al cálculo de los valores característicos, promedios de resistencia última, desviación estándar, coeficiente de variación y número de ensayos, se muestra en la tabla 2 , junto con el percentil 5 y los valores característicos.

Tabla 2. Resistencia última promedio y valores caracteristicos de la Guadua angustifolia del departamento del Huila

\begin{tabular}{c|c|c|c|c}
\hline Datos estadísticos & Inferior & Media & Superior & Total \\
\hline Número de datos (N) & 44 & 44 & 42 & 130 \\
\hline Resistencia última promedio (Mpa) & 36,75 & 36,39 & 40,48 & 37,83 \\
\hline Desviación estándar (s) & 4,77 & 4,60 & 5,03 & 5,11 \\
\hline Coeficiente de variación (\%) & 12,99 & 12,63 & 12,43 & 13,5 \\
\hline Percentil 5 (Mpa) & 28,90 & 28,83 & 32,20 & 29,43 \\
\hline Valor Caracteristico (Mpa) & 27,37 & 27,35 & 30,54 & 28,49 \\
\hline
\end{tabular}

\subsection{Resistencia última a la compresión paralela a la fibra en otras investigaciones}

Se presentan los valores de resistencia última en probetas de guadua angustifolia con relación diámetro/longitud 1:1, en estudios ajustados a las metodologías de la Norma Técnica Colombiana NTC 5525 (tabla 3) y la ISO 22157 (tabla 4).

Tabla 3. Resistencia última a la compresión paralela a la fibra de probetas con relación diámetro/longitud 1:1 de diferentes procedencias (Mpa)

\begin{tabular}{c|c|c|c|c|c}
\hline Sección & $\begin{array}{c}\text { Ghavami y } \\
\text { Marinho (2005) }\end{array}$ & $\begin{array}{c}\text { González } \\
\text { et al (2008) }\end{array}$ & Lozano et al (2010) & Muñoz (2011) & $\begin{array}{c}\text { Capera y Erazo } \\
\text { (2012) }\end{array}$ \\
\hline Inferior & $28,36^{\mathrm{B}}$ & $24,09^{\mathbb{R}}$ & $28,11^{\mathrm{S}}-30,08^{\mathrm{Q}}-29,78^{\mathrm{V}}$ & $42,01^{\mathrm{R}}-37,28^{\mathrm{R}}-41,16^{\mathrm{V}}$ & $36,75^{\mathrm{H}}$ \\
\hline Media & $31,77^{\mathrm{B}}$ & $29,14^{\mathrm{R}}$ & $25,77^{\mathrm{S}}-30,35^{\mathrm{Q}}-32,07^{\mathrm{V}}$ & $73,90^{\mathrm{C}}-39,82^{\mathrm{Q}}-40,43^{\mathrm{R}}$ & $36,39^{\mathrm{H}}$ \\
\hline Superior & $25,27^{\mathrm{B}}$ & $27,54^{\mathrm{R}}$ & $39,78^{\mathrm{S}}-39,15^{\mathrm{Q}}-33,91^{\mathrm{V}}$ & $45,19^{\mathrm{C}}-53,85^{\mathrm{C}}-50,13^{\mathrm{V}}$ & $40,48^{\mathrm{H}}$ \\
\hline Promedio & - & $26,92^{\mathbb{R}}$ & $32,38^{\mathrm{S}}-33,89^{\mathrm{Q}}-32,38^{\mathrm{V}}$ & - & $37,83^{\mathrm{H}}$ \\
\hline
\end{tabular}

Los superindices hacen referencia a muestras de guadua angustifolia de los departamentos de (C) Caldas, (H) Hula, (Q) Quindio, (R) Risaralda, (S) Cundinamarca y (V) Valle del Cauca, se incluye además un estudio realizado fuera del pais (B) PUC-Río.

En la tabla 3 se observa la mayor resistencia obtenida para la guadua angustifolia del departamento del Huila cuando es solicitada a compresión paralela; esta situación se presenta tras excluir los resultados alcanzados por Muñoz, que hacen referencia a clones superiores de guadua angustifolia para la construcción - seleccionada de lugares previamente explorados por García (2004) y González (2006) -. Para confirmar dicha superioridad, conviene mencionar que el contenido de humedad promedio registrado por Lozano et al (2010) fue de $89,08 \%$ y por González et al (2008) entre 12,4 y $60,6 \%$, ninguno de ellos significativamente superior al $86,16 \%$ registrado en este 
estudio. Se comprueba que además del contenido de humedad, la resistencia varía de acuerdo al segmento de culmo analizado, siendo esta mayor en las partes más altas.

González (2006) y Rivera (2008) que implementaron los procedimientos estipulados en la Norma ISO 22157 presentaron los resultados que se muestran en la tabla 4 , el contenido de humedad promedio registrado por el primero fue de $13,60 \%$ y $12 \%$ para el segundo. Estos estudios se centraron en la guadua de los departamentos de Quindio y Caldas, la relación adoptada entre el diámetro y la longitud de la probeta fue de 1:2, se observa como la resistencia cambia significativamente con el contenido de humedad si se compara con los resultados del proyecto tomado como referencia.

Tabla 4. Comparación. Resultados en estudios realizados implementando la ISO 22157.

\begin{tabular}{c|c|c}
\hline Gonzilez (2006) & Rivera (2008) & $\begin{array}{c}\text { Capera y Erazo } \\
(\mathbf{2 0 1 2})\end{array}$ \\
\hline $\begin{array}{c}51,57^{\mathrm{C}} \mathrm{Mpa} \mathrm{y} \\
\text { De } 56,29 \text { a } 59,43^{\mathrm{Q}} \mathrm{Mpa}\end{array}$ & $57,60^{\mathrm{Q}} \mathrm{Mpa}$ & $37,83^{\mathrm{H}} \mathrm{Mpa}$ \\
\hline
\end{tabular}

Los superindices hacen referencia a muestras de guadua angustifolia de los departamentos de (C) Caldas, (H) Hulla y (Q) Quindio.

Lamus (2008), para guadua del eje cafetero, encontró en probetas con la misma relación diámetro/longitud, una resistencia promedio de $36,60 \mathrm{Mpa}$, sin detallar el contenido de humedad, la metodología en las pruebas de laboratorio y los tramos de los culmos utilizados. En esta misma región Garcia (2004) centró su investigación, analizando únicamente la sección superior de los culmos elegidos, obteniendo una resistencia promedio de 31,97 Mpa sin detallar la metodología empleada durante las pruebas de laboratorio y los contenidos de humedad durante las pruebas.

Previo a la publicación de la norma ISO 22157 en el 2004, se realizaron los estudios que arrojaron los siguientes resultados (tabla 5) mencionados por Takeuchi (2004) en compresión paralela a la fibra. Es importante mencionar que López y Silva (2000) realizaron sus ensayos en columnas cortas, y que el contenido de humedad promedio para los ensayos de Martín y Mateus (1981) fue del 12\%, para Martínez (1992) entre 22,66 y 66,67\% y para Durán y Uribe (2002) 18,23\% en probetas relación 1:1.

Tabla 5. Comparación. Primeros estudios realizados en compresión paralela a la fibra.

\begin{tabular}{c|c|c|c|c}
\hline $\begin{array}{c}\text { Martín y Mateus } \\
(\mathbf{1 9 8 1})\end{array}$ & $\begin{array}{c}\text { Martínez } \\
(\mathbf{1 9 9 2})\end{array}$ & $\begin{array}{c}\text { López y Silva } \\
(\mathbf{2 0 0 0 )}\end{array}$ & $\begin{array}{c}\text { Durán y Uribe } \\
(\mathbf{2 0 0 2})\end{array}$ & $\begin{array}{c}\text { Capera y Erazo } \\
(\mathbf{2 0 1 2})\end{array}$ \\
\hline $662^{\mathrm{C}} \mathrm{kg} / \mathrm{cm}^{2}$ & $343^{\mathrm{Q}} \mathrm{kg} / \mathrm{cm}^{2}$ & $439^{\mathrm{C}} \mathrm{kg} / \mathrm{cm}^{2}$ & $504,37^{\mathrm{Q}} \mathrm{kg} / \mathrm{cm}^{2}$ & $37,83^{\mathrm{H}} \mathrm{Mpa}$ \\
\hline
\end{tabular}

Los superindices hacen referencia a muestras de guadua angustifolia de los departamentos de (C) Caldas,

(H) Huila y (Q) Quindio.

\subsection{Valores característicos en otras investigaciones}

Los valores característicos mostrados en la tabla 6 permiten evidenciar con mayor claridad la diferencia existente entre los resultados de los estudios alli referidos. La marcada diferencia entre los valores característicos calculados por González (2006), Rivera (2008) y Lamus (2008), con los obtenidos por Lozano et al (2010) y en el estudio para guadua del departamento del Huila, se debe principalmente a diferencias significativas en el contenido de humedad, recordando que en los últimos se obtuvieron valores de 89,08 y $86,16 \%$ respectivamente, producto de la inmersión en agua de las probetas por un periodo de dos semanas antes de los ensayos de resistencia. 
Ghavami y Marinho (2005), González et al (2008) y Muñoz (2011) no calcularon valores característicos, sin embargo es de esperarse resultados acordes a las comparaciones establecidas a partir de la tabla 3 . Se evidencia nuevamente la superioridad de la guadua angustifolia del departamento del Huila, comparada con la guadua de los departamentos de Cundinamarca, Quindio y Valle del Cauca.

Tabla 6. Comparación. Valores característicos en elementos solicitados a compresión paralela

\begin{tabular}{c|c|c|c|c|c}
\hline Sección & $\begin{array}{c}\text { González } \\
(\mathbf{2 0 0 6}\end{array}$ & Rivera (2008) & Lamus (2008) & Lozano et al (2010) & $\begin{array}{c}\text { Caperay } \\
\text { Erazo (2012) }\end{array}$ \\
\hline Promedio & $40,42^{\bullet Q}$ & $37,70^{\complement}$ & $36,60^{\mathbb{Z}}$ & $20,57^{5}-19,51^{\mathrm{Q}}-20,25^{\mathrm{V}}$ & $28,49^{\mathrm{H}}$ \\
\hline
\end{tabular}

Los superíndices hacen referencia a muestras de guadua angustifolia de los departamentos de (C) Caldas, (E) Eje cafetero, (H) Huila, (Q) Quindio, (S) Cundinamarca y (V) Valle del Cauca.

\section{Conclusiones}

Comparando los resultados de González et al (2008) con los de Lozano et al (2010) y Capera y Erazo (2012), se observa como la resistencia última a la compresión paralela de la guadua angustifolia kunth, puede variar significativamente entre procedencias independientemente del contenido de humedad. Lo anterior se podria relacionar, con los efectos sobre las características internas del material que podrian provocar las condiciones edafológicas y climatológicas propias de cada región o sitio especifico.

A partir del esfuerzo caracteristico, se encuentra que la resistencia a la compresión paralela de la guadua angustifolia del departamento del Huila (28,49 Mpa), es superior a la resistencia presentada por Lozano et al (2010) para gradua de Cundinamarca (20,57 Mpa), Quindio (19,51 Mpa) y Valle del Cauca $(20,25 \mathrm{Mpa})$. Esta comparación se considera como la más acertada, teniendo en cuenta que las dos investigaciones implementaron los mismos procedimientos y además presentaron contenidos de humedad similares.

Se comprueba como los valores característicos permiten observar con mayor claridad, las diferencias que se presentan entre los resultados de resistencia a la compresión paralela a la fibra o cualquier otra propiedad mecánica, al considerar la representatividad de las muestras de estudio (desviación estándar y tamaño de la población).

La relación diámetro/longitud de las probetas y el procedimiento de inmersión en agua de las mismas, previo a la ejecución de ensayos, marcan la diferencia en los resultados de resistencia a la compresión paralela. La implementación de distintas normas técnicas en cada una de las investigaciones, constituye la razón principal por la que se dificulta realizar comparaciones más precisas.

En sintesis, se puede afirmar que la Guadua angustifolia Kunth del departamento del Huila cuando es solicitada a compresión paralela, presenta mejor desempeño que el material procedente de los departamentos de Cundinamarca y los que conforman el eje cafetero, por tal motivo se debe considerar para realizar futuras investigaciones, asi como promover su producción, comercialización y uso.

\section{Referencias bibliográficas}

1. Capera, A., Erazo, W., 2012. Resistencia a la compresión paralela a la fibra y determinación del módulo de elasticidad de la guadua angustifolia del municipio de Pitalito-Huila. Neiva: Universidad Surcolombiana. Facultad de Ingeniería. $145 \mathrm{pp}$.

2. Garcia, J., 2004. Definición de áreas óptimas de calidad de Guadua (Guadua angustifolia Kunth), onientada a satisfacer las necesidades del mercado. Universidad Tecnológica de Pereira. Facultad de Ingeniería Industrial. $118 \mathrm{pp}$.

3. Ghavami, K., Marinho, A., 2005. Propriedades físicas e mecânicas do colmo inteiro do bambu da espécie Guadua angustifolia. Rio de Janeiro: Revista Brasileira de Engenharia Agrícola e Ambiental, v.9, n.1, 107-114 p. 
4. González, C., 2006. Resistencia a la compresión paralela a la fibra de la Guadua angustifolia y deteminación del módulo de elasticidad Bogotá: Universidad Nacional de Colombia. Departamento de Ingenieria Civil y Agrícola. 95 pp.

5. González, H., Montoya, J., Rubiel, J., 2008. Comportamiento de muestras de Guadua angustifolia Kunth con diafragma y sin diafragma sometidas a esfuerzo de compresión. Pereira: Universidad Tecnológica de Pereira. Scientia et Technica, no 38. ISSN 012-1701.

6. Lamus, F., 2008. Calificación de una conexión viga-columna resistente a momento en guadua angustifolia. Bogotá: Universidad Nacional de Colombia. Departamento de Ingeniería Civil y Agricola. 242 pp.

7. López, L., Silva, M., 2000. Comportamiento sismorresistente de estructuras en bahareque. Manizales: Universidad Nacional de Colombia. Citado por: Takeuchi, C., 2004. Comportamiento estuctural de la guadua angustifolia. Uniones en guadua. En: Ingenieria e Investigacion, v.24, n.002. 3.7 pp.

8. Lozano, J., Luna, P., Takeuchi, C., 2010. Validación de la Guadua angustifolia como material estructural para diseño, por el método de esfuerzos admisibles. Bogotá: Universidad Nacional de Colombia. Departamento de Ingeniería Civil y Agricola. $190 \mathrm{pp}$.

9. Martin, J., Mateus, L., 1981. Determinación de la resistencia a compresión paralela a la fibra de guadua de castilla. Bogota: Universidad Nacional de Colombia. Citado por. Takeuchi, C., 2004. Comportamiento estructural de la guadua angustifolia. Uniones en guadua. En: Ingenieria e Investigación, v.24, n.002. 3-7 pp.

10. Martinez, E., 1992. Puentes en do mayor. Congreso mundial de Bambu/Guadua. Citado por: Takeuchi, C., 2004. Comportamiento estructural de la guadua angustifolia. Uniones en guadua. En: Ingeniería e Investigación, v.24, n.002. $3-7 \mathrm{pp}$.

11. Ministerio de Fomento. Gobiemo de España. (n.d). Consultado en febrero de 2013. Disponible en: CURL: http://www.fomento.gob.es/NR/rdonlyres/7E6874BB-AF3B 4B35-B676F9F6A1930B5C/37457/CAPITULOII

12. Muñoz, J., 2011. Diversidad genética, estructura poblacional y selección de clones superiores de Guadua angustifolia Kunth en la eco-región cafetera de Colombia. Tesis de doctorado en ciencia. Palmira: Universidad Nacional de Colombia. $151 \mathrm{pp}$.

13. Rivera, J., 2008. Comportamiento estructural de pórticos en guadua, arriostrados mediante diagonales en guadua. Bogotá: Universidad Nacional de Colombia. Departamento de Ingeniería Civil y Agricola. $252 \mathrm{pp}$.

14. Unibe, M., Durán, A., 2002. Estudio de elementos solicitados a compresión amados por tres Guaduas. Bogotá: Universidad Nacional de Colombia. Departamento de Ingeniería Civil. $125 \mathrm{pp}$. 